PROCEEDINGS OF THE

AMERICAN MATHEMATICAL SOCIETY

Volume 136, Number 8, August 2008, Pages 2675-2681

S 0002-9939(08)09480-X

Article electronically published on April 2, 2008

\title{
A SEPARABLE DEFORMATION OF THE QUATERNION GROUP ALGEBRA
}

\author{
NURIT BARNEA AND YUVAL GINOSAR
}

(Communicated by Martin Lorenz)

\begin{abstract}
The Donald-Flanigan conjecture asserts that for any finite group $G$ and any field $k$, the group algebra $k G$ can be deformed to a separable algebra. The minimal unsolved instance, namely the quaternion group $Q_{8}$ over a field $k$ of characteristic 2 was considered as a counterexample. We present here a separable deformation of $k Q_{8}$. In a sense, the conjecture for any finite group is open again.
\end{abstract}

\section{INTRODUCTION}

In their paper [1], J. D. Donald and F. J. Flanigan conjectured that any group algebra $k G$ of a finite group $G$ over a field $k$ can be deformed to a semisimple algebra even in the modular case, namely where the order of $G$ is not invertible in $k$. A more customary formulation of the Donald-Flanigan (DF) conjecture is by demanding that the deformed algebra $[k G]_{t}$ should be separable; i.e., it remains semisimple when tensored with the algebraic closure of its base field. If, additionally, the dimensions of the simple components of $[k G]_{t}$ are in one-to-one correspondence with those of the complex group algebra $\mathbb{C} G$, then $[k G]_{t}$ is called a strong solution to the problem.

The DF conjecture was solved for different families of groups, using different methods:

- when the group $G$ has a cyclic $p$-Sylow subgroup and $k$ is an algebraically closed field of characteristic $p$ [12;

- when $G$ has a normal abelian $p$-Sylow subgroup in characteristic $p[6$;

- dihedral groups [3] and semi-dihedral groups [2] in characteristic 2;

- reflection groups in any characteristic (with six exceptions) [7, 8, 11.

In [5], it is claimed that the group algebra $k Q_{8}$, where

$$
Q_{8}=\left\langle\sigma, \tau \mid \sigma^{4}=1, \tau \sigma=\sigma^{3} \tau, \sigma^{2}=\tau^{2}\right\rangle
$$

is the quaternion group of order 8 and $k$ is a field of characteristic 2 , does not admit a separable deformation. This result allegedly gives a counterexample to the DF conjecture. However, as observed by M. Schaps, the proof apparently contains an error (see \$7).

Received by the editors April 23, 2007.

2000 Mathematics Subject Classification. Primary 16S80.

(C)2008 American Mathematical Society Reverts to public domain 28 years from publication 
The aim of this paper is to present a separable deformation of $k Q_{8}$, where $k$ is any field of characteristic 2, reopening the DF conjecture. The same approach works for the generalized quaternion group algebras and will be introduced separately.

\section{Preliminaries}

Let $k[[t]]$ be the ring of formal power series over $k$, and let $k((t))$ be its field of fractions. Recall that the deformed algebra $[k G]_{t}$ has the same underlying $k((t))$ vector space as $k((t)) \otimes_{k} k G$, with multiplication defined on basis elements

$$
g_{1} * g_{2}:=g_{1} g_{2}+\sum_{i \geq 1} \Psi_{i}\left(g_{1}, g_{2}\right) t^{i}, \quad g_{1}, g_{2} \in G
$$

and extended $k((t))$-linearly (such that $t$ is central). Here $g_{1} g_{2}$ is the group multiplication. The functions $\Psi_{i}: G \times G \rightarrow k G$ satisfy certain cohomological conditions induced by the associativity of $[k G]_{t}[\underline{4}, \S \S 1,2]$.

Note that the set of equations (2.1) determines a multiplication on the free $k[[t]]$-module $\Lambda_{t}$ spanned by the elements $\{g\}_{g \in G}$ such that $k G \simeq \Lambda_{t} /\left\langle t \Lambda_{t}\right\rangle$ and $[k G]_{t} \simeq \Lambda_{t} \otimes_{k[[t]]} k((t))$. In a more general context, namely over a domain $R$ which is not necessarily local, the $R$-module $\Lambda_{t}$ which determines the deformation, is required only to be flat rather than free [3, §1].

In what follows, we shall define the deformed algebra $[k G]_{t}$ by using generators and relations. These will implicitly determine the set of equations (2.1).

\section{Sketch of the CONSTRuCtion}

Consider the extension

$$
[\beta]: 1 \rightarrow C_{4} \rightarrow Q_{8} \rightarrow C_{2} \rightarrow 1,
$$

where $C_{2}=\langle\bar{\tau}\rangle$ acts on $C_{4}=\langle\sigma\rangle$ by

$$
\begin{array}{rccl}
\eta: & C_{2} & \rightarrow & \operatorname{Aut}\left(C_{4}\right), \\
\eta(\bar{\tau}): & \sigma & \mapsto & \sigma^{3}\left(=\sigma^{-1}\right),
\end{array}
$$

and the associated 2-cocycle $\beta: C_{2} \times C_{2} \rightarrow C_{4}$ is given by

$$
\beta(1,1)=\beta(1, \bar{\tau})=\beta(\bar{\tau}, 1)=1, \quad \beta(\bar{\tau}, \bar{\tau})=\sigma^{2} .
$$

The group algebra $k Q_{8}$ ( $k$ any field) is isomorphic to the quotient $k C_{4}[y ; \eta] /\langle q(y)\rangle$, where $k C_{4}[y ; \eta]$ is a skew polynomial ring [10, $\left.\S 1.2\right]$, whose indeterminate $y$ acts on the ring of coefficients $k C_{4}$ via the automorphism $\eta(\bar{\tau})$ (extended linearly) and where

$$
q(y):=y^{2}-\sigma^{2} \in k C_{4}[y ; \eta]
$$

is central. The above isomorphism is established by identifying $\tau$ with the indeterminate $y$.

Suppose now that $\operatorname{Char}(k)=2$. The deformed algebra $\left[k Q_{8}\right]_{t}$ is constructed as follows.

In 4.1 the subgroup algebra $k C_{4}$ is deformed to a separable algebra $\left[k C_{4}\right]_{t}$ which is isomorphic to $K \oplus k((t)) \oplus k((t))$, where $K$ is a separable field extension of $k((t))$ of degree 2 .

The next step (\$4.2) is to construct an automorphism $\eta_{t}$ of $\left[k C_{4}\right]_{t}$ which agrees with the action of $C_{2}$ on $k C_{4}$ when specializing $t=0$. This action fixes all three 
primitive idempotents of $\left[k C_{4}\right]_{t}$. In that way, we obtain the skew polynomial ring $\left[k C_{4}\right]_{t}\left[y ; \eta_{t}\right]$.

In $₫ 5$ we deform $q(y)=y^{2}+\sigma^{2}$ to $q_{t}(y)$, a separable polynomial of degree 2 in the center of $\left[k C_{4}\right]_{t}\left[y ; \eta_{t}\right]$.

By factoring out the two-sided ideal generated by $q_{t}(y)$, we establish the deformation

$$
\left[k Q_{8}\right]_{t}:=\left[k C_{4}\right]_{t}\left[y ; \eta_{t}\right] /\left\langle q_{t}(y)\right\rangle .
$$

In $\sqrt{6}$ we show that $\left[k Q_{8}\right]_{t}$ as above is separable. Moreover, passing to the algebraic closure $\overline{k((t))}$ we have

$$
\left[k Q_{8}\right]_{t} \otimes_{k((t))} \overline{k((t))} \simeq \bigoplus_{i=1}^{4} \overline{k((t))} \oplus M_{2}(\overline{k((t))}) .
$$

This is a strong solution to the DF conjecture since its decomposition to simple components is the same as

$$
\mathbb{C} Q_{8} \simeq \bigoplus_{i=1}^{4} \mathbb{C} \oplus M_{2}(\mathbb{C}) .
$$

\section{A deformation of $k C_{4}[y ; \eta]$}

4.1. We begin by constructing $\left[k C_{4}\right]_{t}, C_{4}=\langle\sigma\rangle$. Recall that

$$
k C_{4} \simeq k[x] /\left\langle x^{4}+1\right\rangle
$$

by identifying $\sigma$ with $x+\left\langle x^{4}+1\right\rangle$. We deform the polynomial $x^{4}+1$ to a separable polynomial $p_{t}(x)$ as follows.

Let $k[[t]]^{*}$ be the group of invertible elements of $k[[t]]$ and denote by

$$
U:=\left\{1+z t \mid z \in k[[t]]^{*}\right\}
$$

its subgroup of 1 -units (when $k=\mathbb{F}_{2}, U$ is equal to $k[[t]]^{*}$ ).

Let

$$
a \in k[[t]] \backslash k[[t]]^{*}
$$

be a non-zero element, and let

$$
b, c, d \in U(c \neq d),
$$

such that

$$
\pi(x):=x^{2}+a x+b
$$

is an irreducible (separable) polynomial in $k((t))[x]$. Let

$$
p_{t}(x):=\pi(x)(x+c)(x+d) \in k((t))[x] .
$$

Then the quotient $k((t))[x] /\left\langle p_{t}(x)\right\rangle$ is isomorphic to the direct sum $K \oplus k((t)) \oplus$ $k((t))$, where $K:=k((t))[x] /\langle\pi(x)\rangle$. The field extension $K / k((t))$ is separable and of dimension 2 .

Note that $p_{t=0}(x)=x^{4}+1$ and that only lower order terms of the polynomial were deformed. Hence, the quotient $k[[t]][x] /\left\langle p_{t}(x)\right\rangle$ is $k[[t]]$-free and $k((t))[x] /\left\langle p_{t}(x)\right\rangle$ indeed defines a deformation $\left[k C_{4}\right]_{t}$ of $k C_{4} \simeq k[x] /\left\langle x^{4}+1\right\rangle$. The new multiplication $\sigma^{i} * \sigma^{j}$ of basis elements (2.1) is determined by identifying $\sigma^{i}$ with $\bar{x}^{i}:=x^{i}+\left\langle p_{t}(x)\right\rangle$. We shall continue to use the term $\bar{x}$ in $\left[k C_{4}\right]_{t}$ rather than $\sigma$.

Assume further that there exists $w \in k[[t]]$ such that

$$
(x+w)(x+c)(x+d)=x \pi(x)+a
$$


(see Example 4.3). Then $K \simeq\left(\left[k C_{4}\right]_{t}\right) e_{1}$, where

$$
e_{1}=\frac{(\bar{x}+w)(\bar{x}+c)(\bar{x}+d)}{a} .
$$

The other two primitive idempotents of $\left[k C_{4}\right]_{t}$ are

$$
e_{2}=\frac{c(\bar{x}+d) \pi(\bar{x})}{a(c+d)}, \quad e_{3}=\frac{d(\bar{x}+c) \pi(\bar{x})}{a(c+d)} .
$$

4.2. Let

$$
\eta_{t}: k((t))[x] \rightarrow k((t))[x]
$$

be an algebra endomorphism determined by its value on the generator $x$ as follows:

$$
\eta_{t}(x):=x \pi(x)+x+a .
$$

We compute $\eta_{t}(\pi(x)), \eta_{t}(x+c)$ and $\eta_{t}(x+d)$ :

$$
\begin{aligned}
\eta_{t}(\pi(x))=\eta_{t}(x)^{2}+a \eta_{t}(x)+b & =x^{2} \pi(x)^{2}+x^{2}+a^{2}+a x \pi(x)+a x+a^{2}+b \\
& =\pi(x)\left(x^{2} \pi(x)+a x+1\right) .
\end{aligned}
$$

By (4.1),

$$
\eta_{t}(\pi(x))=\pi(x)+x(x+w) p_{t}(x) \in\langle\pi(x)\rangle .
$$

Next,

By (4.1),

$$
\eta_{t}(x+c)=x \pi(x)+x+a+c .
$$

Similarly,

$$
\eta_{t}(x+c)=(x+c)[(x+w)(x+d)+1] \in\langle x+c\rangle .
$$

By (4.5), (4.6) and (4.7), we obtain that $\eta_{t}\left(p_{t}(x)\right) \in\left\langle p_{t}(x)\right\rangle$, and hence $\eta_{t}$ induces an endomorphism of $k((t))[x] /\left\langle p_{t}(x)\right\rangle$, which we continue to denote by $\eta_{t}$. As can easily be verified, the primitive idempotents given in (4.2) and (4.3) are fixed under $\eta_{t}$ :

$$
\eta_{t}\left(e_{i}\right)=e_{i}, \quad i=1,2,3,
$$

whereas

$$
\eta_{t}\left(\bar{x} e_{1}\right)=\eta_{t}(\bar{x}) e_{1}=(\bar{x} \pi(\bar{x})+\bar{x}+a) e_{1}=(\bar{x}+a) e_{1} .
$$

Hence, $\eta_{t}$ induces an automorphism of $K$ of order 2 while fixing the two copies of $k((t))$ pointwise. Furthermore, one can easily verify that

$$
\eta_{t=0}(\bar{x})=\bar{x}^{3} \text {. }
$$

Consequently, the automorphism $\eta_{t}$ of $\left[k C_{4}\right]_{t}$ agrees with the automorphism $\eta(\bar{\tau})$ of $k C_{4}$ when $t=0$. The skew polynomial ring

$$
\left[k C_{4}\right]_{t}\left[y ; \eta_{t}\right]=\left(k((t))[x] /\left\langle p_{t}(x)\right\rangle\right)\left[y ; \eta_{t}\right]
$$

is therefore a deformation of $k C_{4}[y ; \eta]$.

Note that by (4.8), the idempotents $e_{i}, i=1,2,3$ are central in $\left[k C_{4}\right]_{t}\left[y ; \eta_{t}\right]$ and hence

$$
\left[k C_{4}\right]_{t}\left[y ; \eta_{t}\right]=\bigoplus_{i=1}^{3}\left[k C_{4}\right]_{t}\left[y ; \eta_{t}\right] e_{i} .
$$


4.3. Example. The following is an example for the above construction.

Put

$$
a:=\frac{t+t^{2}+t^{3}}{1+t}, b:=1+t^{2}+t^{3}, c:=\frac{1}{1+t}, d:=1+t+t^{2}, w:=t .
$$

These elements satisfy equation (4.1):

$$
\begin{aligned}
& (x+w)(x+c)(x+d)=(x+t)\left(x+\frac{1}{1+t}\right)\left(x+1+t+t^{2}\right) \\
& =x^{3}+\frac{t+t^{2}+t^{3}}{1+t} x^{2}+\left(1+t^{2}+t^{3}\right) x+\frac{t+t^{2}+t^{3}}{1+t}=x \pi(x)+a .
\end{aligned}
$$

The polynomial

$$
\pi(x)=x^{2}+\frac{t+t^{2}+t^{3}}{1+t} x+1+t^{2}+t^{3}
$$

does not admit roots in $k[[t]] /\left\langle t^{2}\right\rangle$; thus it is irreducible over $k((t))$.

\section{A DEFormation of $q(y)$}

The construction of $\left[k Q_{8}\right]_{t}$ will be completed once the product $\bar{\tau} * \bar{\tau}$ is defined. For this purpose the polynomial $q(y)$ (3.2), which determined the ordinary multiplication $\tau^{2}$, will now be developed in powers of $t$.

For any non-zero element $z \in k[[t]] \backslash k[[t]]^{*}$, let

$$
q_{t}(y):=y^{2}+z \bar{x} \pi(\bar{x}) y+\bar{x}^{2}+a \bar{x} \in\left[k C_{4}\right]_{t}\left[y ; \eta_{t}\right] .
$$

The decomposition of (5.1) with respect to the idempotents $e_{1}, e_{2}, e_{3}$ yields

$$
q_{t}(y)=\left(y^{2}+b\right) e_{1}+\left[y^{2}+z a y+c(c+a)\right] e_{2}+\left[y^{2}+z a y+d(d+a)\right] e_{3} .
$$

We now show that $q_{t}(y)$ is in the center of $\left[k C_{4}\right]_{t}\left[y ; \eta_{t}\right]$.

First, the leading term $y^{2}$ is central since the automorphism $\eta_{t}$ is of order 2 . Next, by (4.8), the free term $b e_{1}+c(c+a) e_{2}+d(d+a) e_{3}$ is invariant under the action of $\eta_{t}$ and hence central. It is left to check that the term $z a\left(e_{2}+e_{3}\right) y$ is central. Indeed, since $e_{2}$ and $e_{3}$ are $\eta_{t}$-invariant, then $z a\left(e_{2}+e_{3}\right) y$ commutes both with $\left[k C_{4}\right]_{t}\left[y ; \eta_{t}\right] e_{2}$ and $\left[k C_{4}\right]_{t}\left[y ; \eta_{t}\right] e_{3}$. Furthermore, by orthogonality,

$$
z a\left(e_{2}+e_{3}\right) y \cdot\left[k C_{4}\right]_{t}\left[y ; \eta_{t}\right] e_{1}=\left[k C_{4}\right]_{t}\left[y ; \eta_{t}\right] e_{1} \cdot z a\left(e_{2}+e_{3}\right) y=0,
$$

and hence $z a\left(e_{2}+e_{3}\right) y$ commutes with $\left[k C_{4}\right]_{t}\left[y ; \eta_{t}\right]$.

Consequently, $\left\langle q_{t}(y)\right\rangle=q_{t}(y)\left[k C_{4}\right]_{t}\left[y ; \eta_{t}\right]$ is a two-sided ideal.

Now, as can easily be deduced from (5.1),

$$
q_{t=0}(y)=y^{2}+\bar{x}^{2}=q(y),
$$

where the leading term $y^{2}$ remains unchanged. Then

$$
\left[k Q_{8}\right]_{t}:=\left[k C_{4}\right]_{t}\left[y ; \eta_{t}\right] /\left\langle q_{t}(y)\right\rangle
$$

is a deformation of $k Q_{8}$, identifying $\bar{\tau}$ with $\bar{y}:=y+\left\langle q_{t}(y)\right\rangle$. 


\section{Separability of $\left[k Q_{8}\right]_{t}$}

Finally, we need to prove that the deformed algebra $\left[k Q_{8}\right]_{t}$ is separable. Moreover, we prove that its decomposition to simple components over the algebraic closure of $k((t))$ resembles that of $\mathbb{C} Q_{8}$. By (4.10), we obtain

$$
\left[k Q_{8}\right]_{t}=\bigoplus_{i=1}^{3}\left[k C_{4}\right]_{t}\left[y ; \eta_{t}\right] e_{i} /\left\langle q_{t}(y) e_{i}\right\rangle .
$$

We handle the three summands in (6.1) separately.

By (5.2),

$$
\left[k C_{4}\right]_{t}\left[y ; \eta_{t}\right] e_{1} /\left\langle q_{t}(y) e_{1}\right\rangle \simeq K\left[y ; \eta_{t}\right] /\left\langle y^{2}+b\right\rangle \simeq K *^{f} C_{2} .
$$

The rightmost term is the crossed product of the group $C_{2}:=\langle\bar{\tau}\rangle$ acting faithfully on the field $K=\left[k C_{4}\right]_{t} e_{1}$ via $\eta_{t}$ (4.9), with a twisting determined by the 2-cocycle $f: C_{2} \times C_{2} \rightarrow K^{*}$ :

$$
f(1,1)=f(1, \bar{\tau})=f(\bar{\tau}, 1)=1, \quad f(\bar{\tau}, \bar{\tau})=b .
$$

This is a central simple algebra over the subfield of invariants $k((t))$ [9, Theorem 4.4.1]. Evidently, this simple algebra is split by $\overline{k((t))}$, i.e.

$$
\left[k C_{4}\right]_{t}\left[y ; \eta_{t}\right] e_{1} /\left\langle q_{t}(y) e_{1}\right\rangle \otimes_{k((t))} \overline{k((t))} \simeq M_{2}(\overline{k((t))}) .
$$

(In fact, $K *^{f} C_{2}$ splits already over $k((t))$, since $b$ is a $C_{2}$-norm of a root of the irreducible polynomial $\pi(x)$ and therefore $f$ is cohomologically trivial.)

Next, since $\eta_{t}$ is trivial on $\left[k C_{4}\right]_{t} e_{2}$, the skew polynomial ring $\left[k C_{4}\right]_{t} e_{2}\left[y ; \eta_{t}\right]$ is actually an ordinary polynomial ring $k((t))[y]$. Again by (5.2),

$$
\left[k C_{4}\right]_{t}\left[y ; \eta_{t}\right] e_{2} /\left\langle q_{t}(y) e_{2}\right\rangle \simeq k((t))[y] /\left\langle y^{2}+z a y+c(c+a)\right\rangle .
$$

Similarly,

$$
\left[k C_{4}\right]_{t}\left[y ; \eta_{t}\right] e_{3} /\left\langle q_{t}(y) e_{3}\right\rangle \simeq k((t))[y] /\left\langle y^{2}+z a y+d(d+a)\right\rangle .
$$

The polynomials $y^{2}+z a y+c(c+a)$ and $y^{2}+z a y+d(d+a)$ are separable (since $z a$ is non-zero). Thus, both $\left[k C_{4}\right]_{t}\left[y ; \eta_{t}\right] e_{2} /\left\langle q_{t}(y) e_{2}\right\rangle$ and $\left[k C_{4}\right]_{t}\left[y ; \eta_{t}\right] e_{3} /\left\langle q_{t}(y) e_{3}\right\rangle$ are separable $k((t))$-algebras, and for $i=2,3$,

$$
\left[k C_{4}\right]_{t}\left[y ; \eta_{t}\right] e_{i} /\left\langle q_{t}(y) e_{i}\right\rangle \otimes_{k((t))} \overline{k((t))} \simeq \overline{k((t))} \oplus \overline{k((t))} .
$$

Equations (6.1), (6.2) and (6.3) yield

$$
\left[k Q_{8}\right]_{t} \otimes_{k((t))} \overline{k((t))} \simeq \bigoplus_{i=1}^{4} \overline{k((t))} \oplus M_{2}(\overline{k((t))}),
$$

as required.

\section{Acknowledgement}

We wish to thank M. Schaps for pointing out to us that there is an error in the attempted proof in [5] that the quaternion group is a counterexample to the DF conjecture. Here is her explanation: The given relations for the group algebra are incorrect. Using the notation in pages 166-7 of [5], if $a=1+i, b=1+j$ and $z=i^{2}=j^{2}$, then $a b+b a=i j(1+z)$, while $a^{2}=b^{2}=1+z$. There is a further error later on when the matrix algebra is deformed to four copies of the field, since a non-commutative algebra can never have a flat deformation to a commutative algebra. 


\section{REFERENCES}

[1] J. D. Donald and F. J. Flanigan, A deformation-theoretic version of Maschke's theorem for modular group algebras: The commutative case, J. Algebra 29 (1974), 98-102. MR0342568 (49:7314)

[2] K. Erdmann, On semisimple deformations of local semidihedral algebras, Arch. Math. 63 (1994), no. 6, 481-487. MR.1300746 (95k:16044)

[3] K. Erdmann and M. Schaps, Deformation of tame blocks and related algebras, in: Quantum Deformations of Algebras and Their Representations, Israel Math. Conf. Proc. 7, Bar-Ilan Univ., Ramat Gan (1993), 25-44. MR.1261899 (94m:16036)

[4] M. Gerstenhaber, On the deformation of rings and algebras, Ann. of Math. 79 (1964), 59-103. MR0171807 (30:2034)

[5] M. Gerstenhaber and A. Giaquinto, Compatible deformations, Contemp. Math. 229, Amer. Math. Soc., Providence, RI (1998), 159-168. MR1676217 (2000e:16029)

[6] M. Gerstenhaber and M. E. Schaps, The modular version of Maschke's theorem for normal abelian p-Sylows, J. Pure Appl. Algebra 108 (1996), no. 3, 257-264. MR.1384005 (97e:16063)

[7] M. Gerstenhaber and M. E. Schaps, Hecke algebras, $U_{q} \mathrm{sl}_{n}$, and the Donald-Flanigan conjecture for $S_{n}$, Trans. Amer. Math. Soc. 349 (1997), no. 8, 3353-3371. MR.1390035 (97j:20012)

[8] M. Gerstenhaber, A. Giaquinto and M. E. Schaps, The Donald-Flanigan problem for finite reflection groups, Lett. Math. Phys. 56 (2001), no. 1, 41-72. MR1848165 (2002g:16049)

[9] I. N. Herstein, Noncommutative Rings, published by The Mathematical Association of America, distributed by John Wiley \& Sons, Inc., New York, 1968. MR0227205 (37:2790)

[10] J. C. McConnell and J. C. Robson, Noncommutative Noetherian Rings, John Wiley \& Sons, Ltd., Chichester, UK, 1987. MR934572 (89j:16023)

[11] M. Peretz and M. Schaps, Hecke algebras and separable deformations of dihedral groups, Far East J. Math. Sci. (FJMS) 1 (1999), no. 1, 17-26. MR1686657|(2000i:20009)

[12] M. Schaps, A modular version of Maschke's theorem for groups with cyclic p-Sylow subgroups, J. Algebra 163 (1994), no. 3, 623-635. MR.1265854 (95b:20015)

Department of Mathematics, University of Haifa, Haifa 31905, Israel

Department of Mathematics, University of Haifa, Haifa 31905, Israel

E-mail address: ginosar@math.haifa.ac.il 